\title{
BMJ Open Monitoring of Palliative Care Symptoms and Concerns in Specialized Palliative Home Care Using an Electronic Version of the Integrated Palliative care Outcome Scale (Palli-MONITOR): protocol for a mixed-methods study
}

To cite: Bolzani A,

Ramsenthaler C, Hodiamont F, et al. Monitoring of Palliative Care Symptoms and Concerns in Specialized Palliative Home Care Using an Electronic Version of the Integrated Palliative care Outcome Scale (PalliMONITOR): protocol for a mixedmethods study. BMJ Open 2021;11:e042266. doi:10.1136/ bmjopen-2020-042266

- Prepublication history for this paper is available online. To view these files, please visit the journal online (http://dx.doi. org/10.1136/bmjopen-2020042266).

Received 30 June 2020 Accepted 28 April 2021

D Check for updates

(c) Author(s) (or their employer(s)) 2021. Re-use permitted under CC BY-NC. No commercial re-use. See rights and permissions. Published by BMJ.

For numbered affiliations see end of article.

Correspondence to Dr Anna Bolzani;

Anna.Bolzani@med.unimuenchen.de

\section{ABSTRACT}

Introduction Over the last decades, patient-reported outcome (PRO) measures have been developed to better understand the patient's perspective and enable patientcentred care. In palliative care, the Integrated Palliative care Outcome Scale (IPOS) is recommended as a PRO tool. Its implementation in specialised palliative home care (SPHC) would benefit from an electronic version validated for the setting.

Following the Medical Research Council (MRC) guidance, the study Palli-MONITOR is developing (phase 1) and testing the feasibility (phase 2) of implementing the electronic version of IPOS (eIPOS) in the SPHC setting to inform a cluster-randomised phase 3 trial.

Methods and analysis Palli-MONITOR is a multicentre, sequential mixed-methods, two-phase development and feasibility study. The study consists of four substudies. In phase 1 (MRC development phase), qualitative patient interviews and focus groups with SPHC professionals are used to identify barriers and facilitators of elPOS (substudy I). Substudy II tests the equivalence of eIPOS and IPOS in a crossover randomised controlled trial. Phase 2 (MRC feasibility/piloting phase) includes a quasi-experimental study with two control groups (substudy III), and qualitative interviews as well as focus groups to explore the feasibility and acceptability of the developed intervention (substudy IV).

Qualitative data will be analysed with thematic analysis following the framework approach. Quantitative analysis uses a two-way intraclass correlation coefficients model for the equivalence testing. Quantitative analysis of the quasiexperimental study will focus on the primary outcomes, recruitment rates and completeness of eIPOS. Secondary outcomes will include intraindividual change in palliative symptoms and concerns, quality of life and symptom burden. Ethics and dissemination Approval of the ethics committee of the Ludwig Maximilian University Munich was received for all study parts. Results and experiences will be presented at congresses and in written form. Additionally, participating SPHC teams will receive summarised results.

Trial registration number NCT03879668.
Strengths and limitations of this study

- This exploratory study systematically develops and tests an electronic version of the Integrated Palliative care Outcome Scale, a valid and reliable paper-andpencil patient-reported outcome in palliative care, for the specialist palliative home care setting.

- This protocol follows the Medical Research Council guidance on the development and evaluation of complex interventions which will increase the validity of results.

- Early involvement of stakeholders such as palliative care patients and professionals in the development phase will ensure rich data from multiple perspectives to inform a feasible intervention for this specialised setting.

- Potential sampling bias due to the convenience sample based on staff identification of eligible patients will be limited by training staff and co-designing the intervention with staff from the participating sites.

\section{INTRODUCTION}

In Germany, patients with severe and lifethreatening conditions are entitled under law to receive specialised palliative home care (SPHC) since 2007. ${ }^{1}$ Currently, over 300 SPHC teams are established in Germany, providing multiprofessional palliative care at home for patients with advanced life-limiting diseases and complex symptoms. ${ }^{2-4}$ Besides professional care focusing on symptom control, this also includes psychological and spiritual support as well as coordination between other healthcare professionals, for instance, general physicians. ${ }^{23}$

Over the last decades, patient-reported outcome (PRO) measures have been developed for a better understanding of patient 
needs. They are also used as quality standards for the care received. ${ }^{5}$ However, implementation in healthcare outside of clinical research is still lacking even though feedback in a timely fashion improves patients' outcomes. ${ }^{6}$ Correct interpretation of outcomes, questioning the impact of PROs in healthcare, and limited time for added PRO collection within a busy clinical care setting are mentioned as barriers for wider use..$^{7-9}$ The costs associated with the implementation of the routine use of PROs for patient monitoring are especially considered as a barrier. However, these costs can be reduced by up to $75 \%$ compared with using paper formats. ${ }^{1011}$ The provision and recording of electronic data reduce costs of printing and sending the instruments as well as staff time needed for documentation and administration. ${ }^{12}$

Implementing PROs digitally may facilitate the assessment of patient information and its use in clinical practice, thus improving processes of care. For a successful implementation, electronic PROs (ePROs) need to agree with the users' needs. Poor user-friendliness was a major obstacle for the use of previous PRO systems for patients at the end of life and for patients with sensory or cognitive limitations in old age, thus preventing high and longterm participation and compliance with the self-reported assessment. $^{13}$

Examining the equivalence of ePROs to paper-based versions is still focused mainly on curative populations or cancer populations not at the end of life. ${ }^{11-17}$ Testing the concurrent validity and agreement has yielded moderate to high intraclass correlation coefficients (ICCs) for most patients, but with the notable exception of older adults over 55 years. ${ }^{18} 19$ One recent study established ICCs for test-retest reliability of an ePRO versus its paper-based version for measuring adverse events in patients with curative cancer. ${ }^{11}$ How and whether ePROs show a comparable performance in terms of validity and reliability in a severely ill, older and frail population such as the SPHC population remains to be determined.

In the palliative care setting, the Integrated Palliative care Outcome Scale (IPOS) is recommended as a PRO tool. ${ }^{16}$ IPOS measures palliative care symptoms and concerns of patients with advanced cancer or non-cancer disease and is available for patients and carers. ${ }^{141617}$ The questionnaire consists of 17 items measuring the impact of physical symptoms, emotional and spiritual issues for the patient and family, and information and practical concerns. The questionnaire has been tested in several inpatient and home care settings as a standardised tool to measure current and changing palliative needs of patients. $^{1520}$

In the home care setting, implementation of PRO measures such as IPOS, which are only available in paper format, is challenging. Paper versions cannot easily be transported between team members and patients, resulting in delays in clinical feedback to identified problems. This is detrimental to the frequent monitoring of symptoms necessary for timely and patient-centred care for this vulnerable patient group. A way to monitor patients' perspectives in this setting is the use of ePRO measures. Different tools have been developed in the last years and tested in clinical practice. ${ }^{21}$ However, the potential of PRO monitoring in real time in a palliative care setting is yet to be determined.

The project 'Monitoring of Palliative Care Needs in Specialized Palliative Home Care Using an Electronic Version of the Integrated Palliative care Outcome Scale' (Palli-MONITOR) is developing and testing the implementation of a new electronic version of the previously validated paper-based IPOS (eIPOS). The eIPOS aims to support professional healthcare through standardised collection of patients' perspectives about palliative symptoms and concerns in real time. Palli-MONITOR is designed following the updated Medical Research Council (MRC) guidelines for the development and evaluation of complex interventions, ${ }^{22}$ focusing on the development and the feasibility phase.

The overall aim of Palli-MONITOR is to inform a subsequent cluster-randomised phase 3 trial testing the effects of the developed intervention.

The primary aim of phase 1 (development) is the development of an acceptable and validated eIPOS.

Objectives are:

1. To explore barriers and facilitators of an ePRO as a standardised tool in SPHC.

2. To test the equivalence of the electronic and paper IPOS (patient version).

The primary aim of phase 2 (feasibility/piloting) is testing the feasibility of the planned implementation process.

Objectives are:

1. To evaluate the feasibility of eIPOS use in terms of participant recruitment, data completion, usage rate and technical practicability of the planned intervention.

2. To analyse the presumed change in care processes before and after the implementation of eIPOS.

3. To explore the acceptability of the ePRO measure for patients and SPHC professionals.

\section{METHODS AND ANALYSIS}

Palli-MONITOR is a sequential mixed-methods, twophase development and feasibility study (figure 1). Each of the two project phases consists of two substudies. The results from substudy I and II will inform an acceptable and validated electronic version of eIPOS which will be used in the feasibility study in phase 2 consisting of substudies III and IV. We follow the MRC guidance and use substudies I and II to develop the intervention (MRC phase 1) and substudies III and IV to test the feasibility of the intervention (MRC phase 2). ${ }^{22}$

The project is led by a research team in the Department of Palliative Medicine, University Hospital of the Ludwig Maximilian University (LMU) Munich, supported by two information technology companies. Five SPHC teams participate in Palli-MONITOR based 


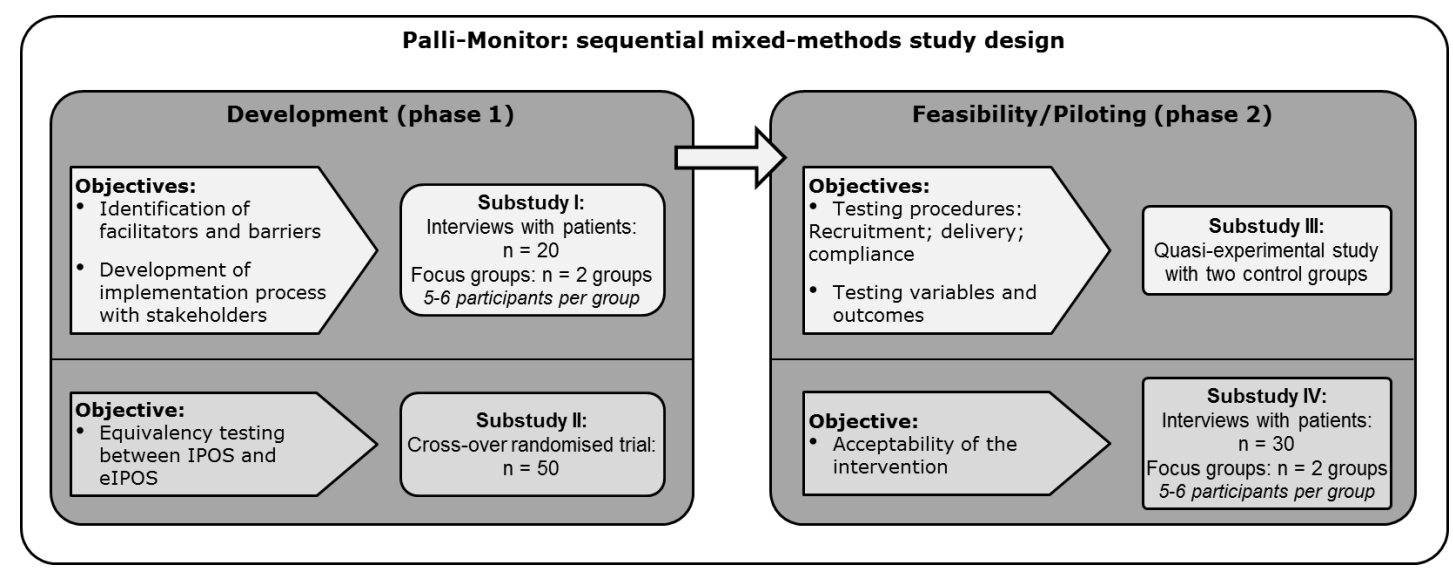

Figure 1 Study design of the project Palli-MONITOR. elPOS, electronic IPOS; IPOS, Integrated Palliative care Outcome Scale.

on the geographical area: two teams provide care in a mostly urban setting, two teams in a rural area and one team in a mixed region. The project is conducted between 2018 and 2021/2022. Substudies III and IV will continue until 2022.

\section{Development of the intervention (MRC phase 1)}

Phase 1 consists of two substudies: for the development of eIPOS, exploring the implementation of eIPOS with stakeholders (substudy I); and testing the equivalence of the developed eIPOS and IPOS (substudy II). Both substudies aim to identify and explore in depth the barriers and facilitators of routinely using an ePRO in a severely ill population and best ways of incorporating this information into everyday practice. Within this development phase, processes and outcomes of implementing eIPOS will be modelled according to implementation science regarding PROs. ${ }^{23-25}$

\section{Substudy I: qualitative interviews and focus groups}

Semistructured interviews with patients and focus groups with SPHC professionals will be conducted. The interview guides will be developed with experienced methodological experts and patients and consist of several topics about the acceptance of electronic monitoring in the SPHC setting (see table 1). The topic guides will be piloted with two members of a patient and public involvement (PPI) group.

Patient interviews will be sampled using a sampling matrix, covering age, gender, residential area (urban or rural) and primary disease to represent potentially different views in the broad variety of patients in SPHC. For recruiting, professionals of the participating SPHC teams will ask eligible patients if they would like to participate in the study.

Patients will be interviewed in their home (or another location chosen by the participant) by one researcher preferably alone to avoid any response bias. If preferred, the participant can choose his or her caregiver to be part of the interview. The interview will be conducted in person and will last around $45-60 \mathrm{~min}$. One focus group will be conducted with the urban teams in the rooms of the Department of Palliative Medicine and one with the rural teams in the rooms of one participating SPHC team due to large distances between the research team and participating teams and limited resources. Focus groups will last about 90-120 min.

Interviews and focus groups will be digitally recorded and transcribed verbatim using anonymisation by a professional transcription office.

\section{Inclusion criteria \\ Patients}

Currently cared for by one of the participating SPHC teams (including access to emergency services) regardless of the underlying disease; $\geq 18$ years; having the capacity to give written consent; not too distressed or ill to participate in the study (assessed by the clinical team); and sufficiently fluent in German.

Table 1 Topic guides for interviews and focus groups

\begin{tabular}{lll}
\hline & Qualitative interviews with patients & Focus groups with professionals \\
\hline $\begin{array}{l}\text { Electronic } \\
\text { monitoring in }\end{array}$ & $\begin{array}{l}\text { General effects of filling in elPOS (for example, } \\
\text { reflecting of own current situation) }\end{array}$ & Support in the identification of current unknown \\
SPHC & Frequency of elPOS use (for example, daily, 2-3 & Ways of implementing results of elPOS in current \\
& times a week, etc) & SPHC \\
Barriers and & Technical challenges & Presentation of patient-reported data \\
facilitators & Motoric issues due to underlying disease & Identification of eligible patients \\
& Design of elPOS & Technical challenges
\end{tabular}

eIPOS, electronic Integrated Palliative care Outcome Scale; SPHC, specialised palliative home care. 


\section{Professionals}

Participants have to be $\geq 18$ years, work in one of the participating SPHC teams and be sufficiently fluent in German.

\section{Recruitment}

\section{Patients}

The participating SPHC teams will identify eligible patients for the interviews. If a patient fulfils the inclusion criteria and has agreed to share their contact details, the SPHC teams will inform the research team. One researcher will then explain the study. If the patient is interested in participating, the researcher will make an appointment for introducing the electronic system to the patient. Before the appointment, the patient will need to give written consent to participate in the study. After the introduction, the patient will test the eIPOS for up to 2 weeks before being interviewed by the same researcher.

\section{Professionals}

Professional members of the participating SPHC teams will be asked to participate in focus groups, consisting of at least one medical and one nursing professional each. Eligible participants will be identified by the research team and/or the contact person(s) of each SPHC team. Written consent will be obtained before participation.

\section{Sample size}

Interviews will be conducted until thematic saturation is reached. $^{26} 27$ Following the sampling criteria aiming at maximal variation, saturation is expected to be achieved after about 20 patient interviews. Two focus groups, with the recommended number of six to nine professionals of the SPHC teams, will be held.

\section{Data analysis}

Data will be analysed with qualitative thematic analysis following the framework approach established by Ritchie and Spencer. ${ }^{28}{ }^{29}$ Two researchers of the project team will independently code the transcripts of five interviews. Preliminary codes will be developed both inductively and deductively based on existing implementation models for PROs in healthcare. ${ }^{23-25}$ Afterwards, the researchers will exchange and reflect their ideas and interpretations of the transcripts. Based on these discussions, one researcher will develop a comprehensive coding framework and arrange identified categories around the major themes. ${ }^{30}$ The focus is the understanding of barriers and facilitators for the use of electronic monitoring in an SPHC setting. Findings shall inform necessary requirements of patients and professional carers to develop a feasible implementation process. A multidisciplinary research team will accompany this process in several meetings to bring up alternative interpretations, give feedback and gain possibilities of reflection and to avoid bias. ${ }^{31}$ The final coding framework will then be applied to the whole data set. MAXQDA will be used to support data management. ${ }^{32}$

Data from this substudy will inform phase 2 (feasibility/ piloting of the intervention) of this project by helping to identify optimal procedures for implementing a selfreport ePRO into routine care. Co-designing the eIPOS intervention with staff members from SPHC services will ensure that eIPOS can help detect the needs of patients, thereby informing care processes and symptom management in routine care.

\section{Substudy II: equivalence testing}

A randomised crossover trial will be conducted to determine the equivalence of eIPOS and IPOS. Participants will complete the two versions of the IPOS in randomised order with a 30-minute waiting period between the questionnaires. The period should be long enough to not complete the second version by memory, but at the same time short enough for symptoms to not change between the two measurement points. The equivalence testing of the electronic version follows the ISPOR guidelines for testing measurement equivalence between electronic and paper-based PRO measures. ${ }^{33}$

To complete the electronic version, participants need to open a website (www.umfrageonline.com/s/pallimonitor) and enter an anonymised ID that will be provided by the researcher. After that, they will complete the questionnaire. The overall appointment lasts about 45-60 min (5-10 min for each version and 30-minute waiting period).

\section{Inclusion criteria}

The inclusion criteria for participants are: currently cared for by one of the participating SPHC teams (including access to emergency services) or in a different palliative setting (palliative care unit or generalist palliative home care) regardless of the underlying disease; $\geq 18$ years; having the capacity to give written consent; not too distressed or ill to participate in the study (as judged by the clinical team); and sufficiently fluent in German.

\section{Recruitment}

Eligible patients will be identified by the participating care teams. If a patient fulfils the criteria and has agreed to share contact details, the research team will be informed. One researcher will then explain the objective, study procedure and requirements in detail to the patient. If the patient is interested, the researcher will make an appointment. Patients on the palliative care unit who fulfil the inclusion criteria are asked by the responsible physician if they would like to take part in this study. Patients will need to give written consent to participate in the study.

\section{Demographic and clinical data collection}

Data collection will take place in the participant's home (or another location chosen by the participant) or in the palliative care unit in the hospital. Preferably, the participant will be alone during the time of testing to avoid any response bias. Participants will be given as much information as needed and the research team will explain the procedure.

In addition to the two IPOS questionnaires, the following sociodemographic and clinical data will be 
collected: age, gender, nationality (German or nonGerman), main diagnosis according to International Classification of Diseases, 10th Revision groups (cancer/ chronic heart disease/lung disease/others), frequency of using electronic devices, type of electronic device (personal computer, notebook, tablet, smartphone, etc), and operating system (Windows, Apple, Android, etc). Furthermore, the research team will measure the time (in minutes) that participants need to complete each IPOS version. After completing the electronic and the paper IPOS, the researcher will ask patients which version they prefer.

\section{Sample size calculation}

With a power of $80 \%$, a target ICC of 0.9 and a significance level of $\alpha=0.05$, the calculated sample size is 47 . Considering possible drop-outs, it is planned to include 50 participants.

\section{Data analysis}

A two-way ICC model with fixed effects will be calculated. ${ }^{34}$ The Wilcoxon test will be used for comparing IPOS values of both versions and completion time. Equivalence will be established for individual IPOS items, for IPOS subscales (physical symptoms subscale, emotional concerns, communication and quality of care subscale $)^{17}$ and the total IPOS score. Bland-Altman plots will be created to present the equivalence and differences between both versions visually for IPOS subscale values and the IPOS total score. To judge the amount of sampling bias, the sociodemographic details of participants will be compared with population profiles of participating sites via descriptive statistics.

Data from substudy II will inform the development of a valid and reliable eIPOS that can be implemented into routine clinical care to be tested in MRC phase 2 of this study.

\section{Feasibility/piloting (MRC phase 2)}

MRC phase 2 of this project focuses on testing procedures of the intervention (piloting), estimating recruitment/ retention rates and determining the acceptability of the intervention (feasibility). Phase 2 consists of two parts (figure 1 for details of the integration within the overall study design): the intervention and implementation procedures developed in phase 1 will be tested (substudy III). Furthermore, the acceptability of the intervention will be determined using a qualitative approach (substudy IV).

Substudy III: quasi-experimental study to pilot and test the feasibility of the elPOS intervention

Substudy III aims to introduce the eIPOS into the routine care processes of each participating SPHC service. In addition to the intervention group receiving eIPOS, there will be two control groups: (a) a prospective control group with patients not receiving the eIPOS, and (b) a retrospective (historical) control group consisting of medical record/chart reviews for patients in the services prior to the introduction of eIPOS. The design of this substudy is summarised in figure 2. All patients cared for by one of the participating SPHC teams are eligible for this study. The intervention consists of patients completing eIPOS for a period of 2 weeks, with corresponding care processes (score review by clinical staff, identifying areas of need and targeting interventions for symptom management accordingly). Participants in the control group, who chose not to use the eIPOS or were ineligible, will not complete any self-report PROs. Care processes for this group will consist of usual care provided by the SPHC service. The retrospective control groups will consist of patients who were cared for by SPHC teams during the last 6 months before the implementation of eIPOS.

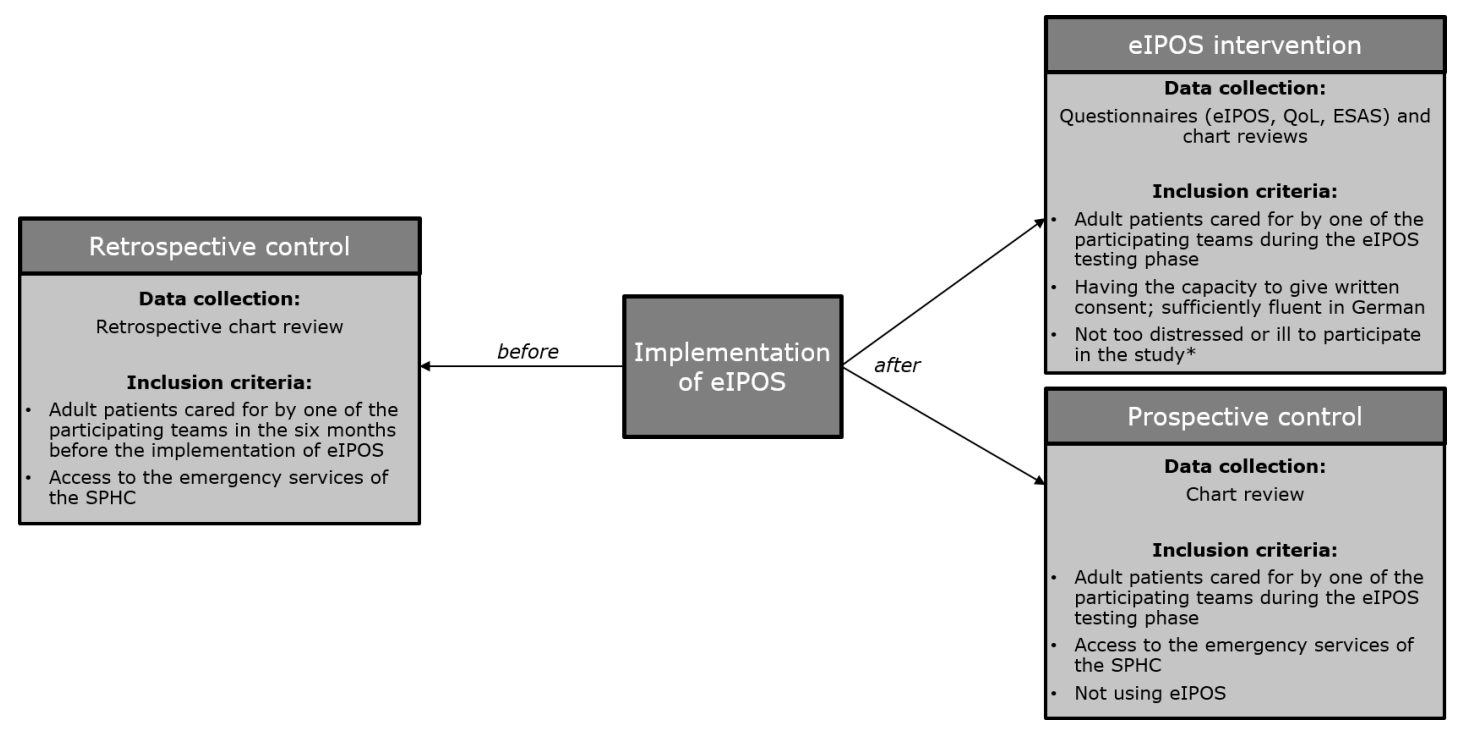

Figure 2 Substudy III-data collection and inclusion criteria of the quasi-experimental study. *Assessed by the clinical team. eIPOS, electronic Integrated Palliative care Outcome Scale; ESAS, Edmonton Symptom Assessment Scale; QoL, Quality of Life; $\mathrm{SPHC}$, specialised palliative home care. 
Table 2 Inclusion criteria for substudy III

\begin{tabular}{lcc}
\hline Intervention group & Prospective control group & Historical control \\
\hline Currently cared for by one of the participating & Currently cared for by & Inclusion/exclusion criteria will be \\
SPHC teams (including access to emergency & one of the participating & finalised after analysing data from phase \\
services) regardless of the underlying disease & SPHC teams (including & 1 \\
Z18 years & access to emergency & Currently planned: inclusion of all \\
Having the capacity to give written consent & services) regardless of the & patients cared for by the participating \\
Not too distressed or ill to participate in the & underlying disease & SPHC teams (including access to \\
study (assessed by the clinical team) & e18 years & emergency services) during the last \\
Sufficiently fluent in German & Not using elPOS & 6 months before the implementation of
\end{tabular}

elPOS, electronic Integrated Palliative care Outcome Scale; SPHC, specialised palliative home care.

\section{Intervention group}

At baseline, a study nurse will introduce the patient to eIPOS. After that, the participant will complete eIPOS every 3 days. There will be no obligation to answer each item of the eIPOS. Standardised procedures (for example: frequency of measurement, time point of introduction) will be finalised after phase 1 . The information from selfreported eIPOS will be used in routine care processes of the service.

\section{Prospective control}

All patients who are cared for by one of the participating SPHC teams, but do not use the eIPOS, will serve as a control group. No additional interventions are planned for this group. However, care processes for this prospective control group might change because of staff being introduced to training around outcome measurement.

\section{Retrospective control}

A retrospective chart review will be conducted to provide information on care processes in the services before the introduction of a self-report PRO measure.

\section{Inclusion criteria}

The inclusion criteria are similar to substudy I and summarised in table 2.

\section{Recruitment}

Intervention group: patients will be recruited from each participating team with the support from a study nurse. A study nurse will inform eligible patients in detail about the aims, study procedure and requirements. If the patient is interested, the study nurse will make an appointment for the introduction of the electronic system and perform the baseline assessment. The patient will need to give written consent before participation.

\section{Outcome measures}

Recruitment as well as drop-out rates, technical feasibility (completeness of each eIPOS) and the influence of eIPOS on provided care will be measured as the primary outcomes of interest.

Secondary outcomes will include change in palliative concerns, quality of life and symptom burden measured with the eIPOS, the EORTC QLQ-C15-PAL (measuring health-related quality of life of palliative patients with cancer $)^{35}$ and the Edmonton Symptom Assessment Scale (assessing symptoms of palliative care patients) ${ }^{36}$ at baseline and after 2 weeks.

Data extracted from medical records for both the intervention and control groups will include the number and severity of palliative symptoms and concerns and the steps taken by professionals for reducing the symptoms. Additionally, the number of unplanned hospital admissions will be collected.

\section{Sample size calculation}

As sample size calculation in feasibility studies should not be based on the primary outcomes of interest, ${ }^{37}$ the intraindividual comparison of quality of life between baseline and 2-week assessment was used as the basis for the estimation. An assumed small effect of $d=0.20$ (with a minimal clinical difference of 4-9 for the EORTC QLQ scale), power $=80 \%$ and $\alpha=0.05$ for a Wilcoxon test with paired data lead to a sample size of 164 . Based on an assumed drop-out rate of $30 \%$, it is aimed to include 213 participants.

For a better understanding of the feasibility of the sample size, and the possible success of the recruitment plan, the total number of patients cared for by the participating SPHC teams ( 1.275 patients/year) will be extracted as well. The control groups will include all patients cared for in the time period explained above.

\section{Data analysis}

Quantitative analysis will include descriptive analysis of the user rate of eIPOS, recruitment and drop-out rate as well as analysis of the technical feasibility based on completeness of eIPOS and number of missing values. Intraindividual changes in the secondary outcomes before and after the implementation of eIPOS will be explored using non-parametric tests (either Wilcoxon or Friedman). Number of symptoms and amount of provided care before and after the implementation of eIPOS will be compared using McNemar tests and Cochran tests for changes in proportions. 
Substudy IV: acceptability of elPOS (feasibility)

The methods and procedures will be identical with substudy I. In short, semistructured interviews with patients and focus groups with SPHC professionals will be conducted, focusing on the experience with eIPOS and the influence of it on the received/provided care.

\section{Inclusion criteria}

Participants who used the eIPOS in substudy III are eligible for the interviews. Apart from that, inclusion criteria are identical with the inclusion criteria of substudy I. Additional inclusion and exclusion criteria may be defined after analysing data of substudy I.

\section{Recruitment}

Recruiting participants for this part will be identical with the approach in substudy I.

\section{Sample size}

The same approach for the number of included participants will be followed as in substudy I.

\section{Data analysis}

The analysis will be identical with substudy I, following the framework approach established by Ritchie and Spencer. ${ }^{28}{ }^{29}$ The analysis will focus on the experiences and opinions of patients and professionals on the implementation of the eIPOS and the influence on the received care.

\section{Data management}

Contact information of patients will only be shared with the research team if patients agreed to do so.

Qualitative data (I, IV): an external company will transcribe the interviews and anonymise them in the process. This company has to comply with data protection and confidentiality regulations. Audio recordings will be deleted once the transcript is provided. Additional data (for example, age and gender) will be collected anonymously and be linked to the transcript with a random study ID.

Quantitative data (II, III): data from medical records will be extracted anonymously. Original data with personal information will only be visible to study nurses who are part of the SPHC teams. Researchers will not have any access to personal patient information. Quantitative data will be saved for 10 years on a secured server at the LMU Munich.

\section{Adverse events}

Even though adverse events are judged to be unlikely for this intervention, all study nurses and research team members will follow a distress protocol and report any adverse events associated with the intervention. Researchers and study nurses are asked to note any reasons for drop-outs and withdrawals during the course of this project.

\section{Patient and public involvement}

PPI in research is of particular importance, especially in palliative care with a vulnerable group of patients. ${ }^{38} \mathrm{In}$
Palli-MONITOR, a PPI group will be involved in all stages of the study to take the perspective of stakeholders and lay persons into account. ${ }^{39}$ Adults with advanced diseases, relatives of palliative patients, voluntary hospice workers and professional carers will be part of this group.

The group members will be involved in the development of documents, such as participant information or interview guides. Furthermore, progress of the study, unexpected challenges and aspects of research ethics from the perspective of those potentially affected by the research will be discussed.

\section{Strengths and limitations}

In this study, the research team will work with a variety of stakeholders to consider different requirements, perspectives and opinions in this very challenging research setting. A mixed-methods approach was chosen to explore different barriers and facilitators of the planned intervention.

Recruitment is a natural challenge in the palliative care setting. Even more so, patients eligible for SPHC suffer from life-limiting diseases with a complex symptom burden. Developing a rapid process for the identification and inclusion of eligible patients with the participating SPHC teams as well as the fact that all study appointments will take place in the patients' home should increase the recruitment rates for all study parts. A limitation of the study is its convenience sample based on staff identification of eligible patients in participating study sites. Therefore, gatekeeping and sampling of only comparatively fitter patients cannot be ruled out completely. We hope to mitigate this sampling bias by training staff and co-designing the intervention with staff from the participating sites. We will compare the sociodemographic details of recruited patients with population data from the services to quantify this possible bias.

The use in clinical practice will depend on the compliance with the newly developed intervention. ${ }^{40}$ Other researchers, for instance, Lang et $a l^{41}$ explored noncompliance with a telemedical intervention for multimorbid patients over 65 years and discovered that missing additional benefits of the intervention, missing content variety and no interest in telemedical care were the main reasons for drop-outs and/or not using the intervention at all. Studies with patients suffering from heart failure or chronic obstructive pulmonary disease testing telemedical interventions have a drop-out rate of around $20 \%$ and almost one-third of the patients refuse to participate. ${ }^{42}$

Therefore, the involvement of patients and professionals is crucial in the development and implementation of eIPOS. In phase 1, barriers and facilitators of the planned intervention will be explored. The results will inform the development of the implementation process. There will also be training sessions on site for each SPHC team between phase 1 and 2 to present results, and to discuss positive as well as negative comments received in phase 1 . 
Results of this project will influence the preparation and conduct of a planned randomised phase 3 study to determine the effect of the developed intervention.

\section{ETHICS AND DISSEMINATION}

Approval of the research ethics committee of the LMU Munich for all study parts was received (18-815; 18-871; $19-512 ; 19-586$; 19-585).

Any changes of this protocol that will be made during the conduct of this project will be discussed in further publications. Besides presenting results and experiences at congresses and in written form, each participating SPHC team will receive a summary of the results.

Due to different healthcare structures and the concept of the SPHC related to the German context, some of the results may be published in German journals.

\section{Author affiliations}

${ }^{1}$ Department of Palliative Medicine, University Hospital, LMU Munich, Munich, Germany

${ }^{2}$ Department of Palliative Medicine, Albert Ludwig University of Freiburg, Freiburg im Breisgau, Germany

${ }^{3}$ Cicely Saunders Institute, Department of Palliative Care, Policy and Rehabilitation, King's College London, London, UK

Contributors $\mathrm{CB}$ is the chief investigator and responsible party for the design of the study. $A B$ is the coordinating investigator and responsible for the conduct of this study. $C B, F H, C R$ and $A B$ conceptualised the study. $A B$ drafted the protocol manuscript. ISB-F supported the development of the manuscript. All authors provided critical feedback to the manuscript, read and approved the final draft.

Funding This work was supported by Federal Joint Committee German Innovation Fund (Innovationsausschuss des Gemeinsamen Bundesausschuss), grant number 01VSF17014.

Competing interests None declared.

Patient and public involvement Patients and/or the public were involved in the design, or conduct, or reporting, or dissemination plans of this research. Refer to the Methods section for further details.

Patient consent for publication Not required.

Provenance and peer review Not commissioned; externally peer reviewed.

Open access This is an open access article distributed in accordance with the Creative Commons Attribution Non Commercial (CC BY-NC 4.0) license, which permits others to distribute, remix, adapt, build upon this work non-commercially, and license their derivative works on different terms, provided the original work is properly cited, appropriate credit is given, any changes made indicated, and the use is non-commercial. See: http://creativecommons.org/licenses/by-nc/4.0/.

ORCID iD

Anna Bolzani http://orcid.org/0000-0002-3049-9805

\section{REFERENCES}

1 Gemeinsamer Bundesausschuss (G-BA). Richtlinie des Gemeinsamen Bundesausschusses Zur Verordnung von spezialisierter ambulanter Palliativversorgung (spezialisierte ambulante Palliativversorgungs-Richtlinie/SAPV-RL), 2007.

2 Alt-Epping B, Nauck F. Spezialisierte Ambulante Palliativversorgung (SAPV). Bundesgesundheitsblatt - Gesundheitsforschung Gesundheitsschutz 2015;58:430-5.

3 Groh G, Vyhnalek B, Feddersen B, et al. Effectiveness of a specialized outpatient palliative care service as experienced by patients and caregivers. J Palliat Med 2013;16:848-56.

4 Kassenärztliche Bundesvereinigung. Spezialisierte Ambulante Palliativversorgung, 2017. Available: http://gesundheitsdaten.kbv.de/ $\mathrm{cms} / \mathrm{html} / 16744 . \mathrm{php}$
5 Schneider W, Eschenbruch N, Thoms U, et al. Wirksamkeit und Qualitätssicherung in Der SAPV-Praxis-Eine explorative Begleitstudie. Ergebnisbericht, 2011.

6 Currow DC, Allingham S, Yates P, et al. Improving national hospice/palliative care service symptom outcomes systematically through point-of-care data collection, structured feedback and benchmarking. Support Care Cancer 2015;23:307-15.

7 Dunckley M, Aspinal F, Addington-Hall JM, et al. A research study to identify facilitators and barriers to outcome measure implementation. Int J Palliat Nurs 2005;11:218-25.

8 Noonan VK, Lyddiatt A, Ware P, et al. Montreal Accord on PatientReported Outcomes (PROs) use series - paper 3: patient-reported outcomes can facilitate shared decision-making and guide selfmanagement. J Clin Epidemiol 2017;89:125-35.

9 Radionova N, Becker G, Mayer-Steinacker R, et al. The views of physicians and nurses on the potentials of an electronic assessment system for recognizing the needs of patients in palliative care. BMC Palliat Care 2020;19:1-9.

10 Basch E, lasonos A, Barz A, et al. Long-term toxicity monitoring via electronic patient-reported outcomes in patients receiving chemotherapy. J Clin Oncol 2007;25:5374-80.

11 Bennett AV, Jensen RE, Basch E. Electronic patient-reported outcome systems in oncology clinical practice. CA Cancer J Clin 2012;62:337-47.

12 Kotronoulas G, Kearney N, Maguire R, et al. What is the value of the routine use of patient-reported outcome measures toward improvement of patient outcomes, processes of care, and health service outcomes in cancer care? A systematic review of controlled trials. J Clin Oncol 2014;32:1480-501.

13 Velikova G, Booth L, Smith AB, et al. Measuring quality of life in routine oncology practice improves communication and patient wellbeing: a randomized controlled trial. $J$ Clin Oncol 2004;22:714-24.

14 Bausewein C, Daveson BA, Currow DC, et al. EAPC White Paper on outcome measurement in palliative care: Improving practice, attaining outcomes and delivering quality servicesRecommendations from the European Association for Palliative Care (EAPC) Task Force on Outcome Measurement. Palliat Med 2016;30:6-22.

15 Murtagh FE, Ramsenthaler C, Firth A, et al. A brief, patient- and proxy-reported outcome measure in advanced illness: validity, reliability and responsiveness of the integrated palliative care outcome scale (IPOS). Palliat Med 2019;33:1045-57.

16 Onkologie Leitlinienprogramm. Palliativmedizin für Patienten MIT einer nicht heilbaren Krebserkrankung, Langversion 1.1, 2015.

17 Schildmann EK, Groeneveld El, Denzel J, et al. Discovering the hidden benefits of cognitive interviewing in two languages: the first phase of a validation study of the integrated palliative care outcome scale. Palliat Med 2016;30:599-610.

18 Marcano Belisario JS, Jamsek J, Huckvale K, et al. Comparison of self-administered survey questionnaire responses collected using mobile apps versus other methods. Cochrane Database Syst Rev 2015;7:Mr000042.

19 Muehlhausen W, Doll H, Quadri N, et al. Equivalence of electronic and paper administration of patient-reported outcome measures: a systematic review and meta-analysis of studies conducted between 2007 and 2013. Health Qual Life Outcomes 2015;13:167.

20 MySupport. MySupport: Implementierung und evaluation einer routinemäßigen Erfassung von patient-centered outcome measures (PCOM) in onkologischen und palliativen Versorgungskontexten, 2019. Available: https://www.drks.de/drks_web/navigate.do? navigationld=trial. $H T M L \& T R I A L \_I D=D R K S 00016681$

21 Castelnuovo G, Mauri G, Simpson S, et al. New technologies for the management and rehabilitation of chronic diseases and conditions. Biomed Res Int 2015;2015:180436.

22 et alCraig P, Dieppe P, Maclntyre S. Developing and evaluating complex interventions: new guidance 2019. Available: https://mrc. ukri.org/documents/pdf/complex-interventions-guidance/ [Accessed 14 Apr 2020].

23 Antunes B, Harding R, Higginson IJ, et al. Implementing patientreported outcome measures in palliative care clinical practice: a systematic review of facilitators and barriers. Palliat Med 2014;28:158-75.

24 Greenhalgh J. The applications of PROs in clinical practice: what are they, do they work, and why? Qual Life Res 2009;18:115-23.

25 Greenhalgh T, Abimbola S. The NASSS framework - a synthesis of multiple theories of technology implementation. Stud Health Technol Inform 2019;263:193-204.

26 Przyborski A, Wohlrab-Sahr M. Qualitative Sozialforschung. ein Arbeitsbuch: Oldenburg Wissenschaftsverlag, 2014.

27 Morse JM. Sampling in grounded theory. The SAGE handbook of grounded theory, 2010: 229-44. 
28 Ritchie J, Spencer L. Qualitative data analysis for applied policy research. analyzing qualitative data. Routledge, 2002: 173-94.

29 Schreier M. Qualitative content analysis in practice. Sage publications, 2012

30 Rädiker S, Kuckartz U. Analyse qualitativer Daten MIT MAXQDA, 2019.

31 Hopf C. Schriften zu Methodologie und Methoden qualitativer Sozialforschung: Herausgegeben von Wulf Hopf und Udo Kuckartz. Springer-Verlag, 2016

32 Kuckartz U, Rädiker S. Datenaufbereitung und Datenbereinigung in Der qualitativen Sozialforschung. Handbuch Methoden Der empirischen Sozialforschung. Springer, 2019: 441-56.

33 Coons SJ, Gwaltney CJ, Hays RD, et al. Recommendations on evidence needed to support measurement equivalence between electronic and paper-based patient-reported outcome (PRO) measures: ISPOR ePRO good research practices task force report. Value Health 2009;12:419-29.

34 Gwaltney CJ, Shields AL, Shiffman S. Equivalence of electronic and paper-and-pencil administration of patient-reported outcome measures: a meta-analytic review. Value Health 2008;11:322-33.

35 Groenvold M, Petersen MA, Aaronson NK, et al. The development of the EORTC QLQ-C15-PAL: a shortened questionnaire for cancer patients in palliative care. Eur J Cancer 2006;42:55-64.
36 Bruera E, Kuehn N, Miller MJ, et al. The Edmonton symptom assessment system (ESAs): a simple method for the assessment of palliative care patients. J Palliat Care 1991;7:6-9.

37 Avery KNL, Williamson PR, Gamble C, et al. Informing efficient randomised controlled trials: exploration of challenges in developing progression criteria for internal pilot studies. BMJ Open 2017;7:e013537.

38 Daveson BA, de Wolf-Linder S, Witt J, et al. Results of a transparent expert consultation on patient and public involvement in palliative care research. Palliat Med 2015;29:939-49.

39 Wright M, Kilian H, Block M, et al. Partizipative Qualitätsentwicklung: Zielgruppen in alle Phasen Der Projektgestaltung einbeziehen. Das Gesundheitswesen 2015;77:S141-2.

40 Dockweiler C. Akzeptanz Der Telemedizin. eHealth in Deutschland. Springer, 2016: 257-71

41 Lang C, Scheibe M, Voigt K, et al. Motive für die Nichtakzeptanz und Nichtnutzung einer Telemonitoring-Anwendung Im häuslichen Umfeld durch multimorbide Patienten über 65 Jahre. Zeitschrift für Evidenz, Fortbildung und Qualität im Gesundheitswesen 2019;141:76-88.

42 Gorst SL, Armitage CJ, Brownsell S, et al. Home telehealth uptake and continued use among heart failure and chronic obstructive pulmonary disease patients: a systematic review. Ann Behav Med 2014;48:323-36. 